\title{
Lipid parameters and vitamin A modify cardiovascular risk prediction by plasma neopterin
}

Indu Dhar ${ }^{1}$, Sumia Siddique ${ }^{2}$, Eva R Pedersen ${ }^{3}$, Gard FT Svingen ${ }^{3}$, Vegard Lysne ${ }^{1,3}$, Thomas Olsen $^{4}$, Dennis W Nilsen ${ }^{5,6}$, Jan Erik Nordrehaug ${ }^{5,6}$, Øivind Midttun ${ }^{7}$, Per M Ueland ${ }^{7}$, Grethe S. Tell ${ }^{8,9}$, Ottar K Nygård ${ }^{1,3}$

${ }^{1}$ Centre for Nutrition, Department of Clinical Science, University of Bergen, Bergen, N-5021 Norway

${ }^{2}$ Faculty of Medicine, University of Bergen, Bergen N-5021, Norway

${ }^{3}$ Department of Heart Disease, Haukeland University Hospital, Bergen N-5021, Norway,

${ }^{4}$ Department of Nutrition, Institute of Basic Medical Sciences, University of Oslo, Oslo 0372

Norway

${ }^{5}$ Department of Cardiology, Stavanger University Hospital, Stavanger 4011, Norway,

${ }^{6}$ Department of Clinical Science, University of Bergen, Bergen N-5021, Norway

${ }^{7}$ Bevital AS, Bergen N-5021 Norway

${ }^{8}$ Department of Global Public Health and Primary Care, University of Bergen, Bergen N5020, Norway

${ }^{9}$ Division of mental and physical health, Norwegian Institute of Public Health, Bergen N-5808 Norway

Address for correspondence: Indu Dhar $\mathrm{PhD}$, Centre for Nutrition, Department of Clinical Science, University of Bergen, Laboratory Building, 9th floor, Haukeland University Hospital, Jonas Lies vei 87, Bergen N-5021; phone +47 40982645; e-mail: Indu.Dhar@uib.no

Total word count (main manuscript excluding references, tables and figures): 2898 


\begin{abstract}
Objectives- Oxidized cholesterol metabolites are linked to increased production of the active vitamin A (Vit-A) form and monocyte/macrophage activation, which may be reflected by neopterin, a marker of both interferon- $\gamma$ - mediated immune activation and coronary artery disease risk. We examined the influence of serum lipid parameters and Vit-A on the risk association between neopterin and incident acute myocardial infarction (AMI).
\end{abstract}

Methods- We included 4130 patients with suspected stable angina pectoris (SAP), of whom $80 \%$ received lipid-lowering treatment with statins. Risk associations between plasma neopterin and AMI are given as HRs per SD increase in log-transformed neopterin. Results- During a median follow-up of 7.5 years, 530 (12.8\%) patients experienced an AMI. In age and sex-adjusted analysis, plasma neopterin was positively associated with incident AMI (HR [95\% CI] per SD: 1.26 [1.17-1.35]). However, the estimates were most pronounced in patients with serum low-density lipoprotein-cholesterol (LDL-C) or apolipoprotein (apo) B100 below-median (HR [95\% CI] per SD: 1.35 [1.24-1.48] and 1.42[1.27-1.58], respectively; both $P_{\text {interaction }} \leq 0.03$ ). We also observed a particularly strong risk association in those with above-median Vit-A (HR [95\% CI] per SD: 1.32 [1.21-1.44]; $P_{\text {interaction }}=0.03$ ). The estimates were slightly modified after multivariable adjustment.

Conclusions- In patients with suspected SAP, the majority of whom receiving statin therapy, high plasma neopterin was associated with increased risk of AMI particularly among those with low LDL-C and apoB100 or high Vit-A levels. The particularly strong relationship of plasma neopterin with residual cardiovascular risk in patients with low lipid levels should be further investigated.

Key words-Acute myocardial infarction, neopterin, lipids, immune system 


\section{Key questions}

\section{What is already known on this topic}

- Higher plasma neopterin, an interferon- $\gamma$ (IFN) - mediated immune activation marker is associated with increased risk of cardiovascular events

- Evidence suggests that alterations in lipid metabolism influences cellular immune activation and are a major contributing factor to the development of atherosclerotic cardiovascular diseases.

- On the other hand, statins, aimed at improving the lipid profile, do not eliminate atherosclerosis, as an incident major cardiovascular event occurs in a substantial proportion of patients who receive statin therapy

\section{What this study adds}

- Plasma neopterin was associated with increased risk of incident AMI predominantly in patients with high serum Vit-A and those with low serum LDL-C and apoB100 levels, in whom a particularly strong relationship was suggested among the majority receiving statin therapy.

\section{How might this impact on clinical practice?}

Future studies should investigate whether the risk relationship between plasma neopterin and incident AMI is primarily due to an impaired cholesterol output from liver. 


\section{Introduction}

Atherosclerosis is a multifactorial disease characterized by the accumulation of lipids and chronic inflammation in the arterial wall, and is the major cause of cardiovascular disease (CVD)[1]. Elevated serum low-density lipoprotein-cholesterol (LDL-C) has been established as a major risk factor for atherosclerotic CVD[2]. LDL-C lowering therapy with statins significantly reduces the risk of cardiovascular events[2] and is expected to be caused by an increased uptake[2] and catabolism of atherogenic lipids in the liver[3], suggesting that LDL$\mathrm{C}$ is a cause of atherosclerotic disease[2]. However, residual cardiovascular risk remains prevalent in a substantial proportion of patients on lipid-lowering therapy with statins[4], reflecting the complex pathophysiology of atherosclerotic disease.

Neopterin is a pteridine derivative secreted from activated macrophages and a marker of interferon- $\gamma$ (IFN) - mediated immune activity[5, 6]. Elevated circulating neopterin levels have been associated with adverse cardiovascular events in the subsample of the current cohort[6] and in patients undergoing angiography[7]as well as in healthy, elderly adults[8]. Available evidence suggests a mutual interplay between lipid metabolism and cellular immune activation. More specifically, high plasma neopterin has been associated with reduced LDL-C and high-density lipoprotein cholesterol (HDL-C)[7]. Further, lipid-lowering therapy with peroxisome proliferator-activated receptor (PPAR) $\alpha$ agonists enhances verylow-density lipoprotein (VLDL) apolipoprotein (apo) B100 catabolism and reduces VLDL apoB100 production[9], in addition to impairing the transcription of glycine $\mathrm{N}$ methyltransferase (GNMT)[10], a critical regulator of VLDL assembly and secretion[11]. Notably, these disturbances are classical components of intracellular hepatic lipid accumulation[11, 12], characterized by the secretion of large buoyant triglyceride (TG)-rich but cholesterol poor lipoproteins, resulting in the formation of small-dense LDL (sdLDL) particles which are prone to oxidation[12-14], and thus considered particularly atherogenic 
[13]. Oxidized lipids regulate recruitment and activation of monocytes [1, 15], and lipid oxidation products regulate synthesis of all-trans retinoic acid[16], an active form of vitaminA (Vit-A) involved in the monocyte differentiation[17]. Since monocytes/macrophages involved in atherosclerosis development produce neopterin in response to IFN- $\gamma$ stimulation [5, 6], cellular cholesterol metabolism and transport in macrophages may be reflected in neopterin production. Of note, neopterin was recently shown to enhance cholesterol efflux and suppress foam-cell formation in human monocyte-derived macrophages, and reduce atherosclerosis development in mice[18].

Taken together, these observations suggest that altered lipid metabolism may affect IFN- $\gamma$ mediated cellular immune activation, and thereby possibly modulate CAD risk. We investigated the effect modification by serum lipid measures and Vit-A on the association between systemic neopterin and risk of incident acute myocardial infarction (AMI) in a large cohort of patients with suspected stable angina pectoris (SAP). Since the majority of patients in this study population were treated with lipid-modifying statins, we also evaluated the potential interaction by statin treatment. 


\section{Methods}

\section{Data sources}

The study population has been previously described[19]. In brief, 4164 patients undergoing coronary angiography for suspected SAP during 2000-2004 at Haukeland or Stavanger University Hospitals in Western Norway were studied. Of these, 2573 were enrolled in the Western Norway B-vitamin intervention trial (WENBIT) (ClinicalTrials.gov Identifier: NCT00354081). Subjects with missing data on plasma neopterin $(n=34)$ were excluded, leaving 4130 subjects eligible for the final analyses. The study was performed according to the Declaration of Helsinki, and was approved by the Regional Medical and Health Ethics committee, the Norwegian Medicines Agency and the Norwegian Data Inspectorate. All participants provided written informed consent.

\section{Baseline variables}

Information about medical history was obtained through self-administered questionnaires and subsequently checked against hospital records, as previously reported[19]. Smoking status was defined according to self-reported smoking habits and also included those who reported having quit smoking within $<1$ month prior to examination and patients with serum cotinine $>85 \mathrm{nmol} / \mathrm{L}$.

\section{Biochemical analyses}

Previous reports have described the collection and storage of blood samples and the biochemical analyses of biomarkers[6, 19]. Plasma neopterin was analyzed by highperformance liquid chromatography/tandem mass spectrometry (LC-MS/MS) at BEVITAL AS, Bergen, Norway (www.bevital.no).

\section{End points and follow up}


Data on study events were collected from the Cardiovascular Disease in Norway project (CVDNOR; https://cvdnor.b.uib.no/)[20]. The primary end point was AMI (including fatal and non-fatal) and classification of events have been described previously[6]. Study patients were followed from enrollment until experiencing an AMI or throughout 2009.

\section{Statistical methods}

Continuous variables are presented as medians $\left(25\right.$ th $-75^{\text {th }}$ percentiles $)$ and categorical variables as counts (percentages). Patients' baseline characteristics across plasma neopterin quartiles were tested by linear regression or logistic regression for continuous and categorical variables, respectively.

Cox proportional regression models were used to calculate hazard ratios (HRs) and 95\% confidence intervals (95\% CIs) for incident AMI for plasma neopterin. HRs were reported according to per 1 standard deviation (SD) increment in log-transformed plasma neopterin. The simple survival model (Model 1) was adjusted for age (continuous) and sex. Additional covariates in the multivariable model (Model 2) included body mass index (continuous), current smoking (yes/no), hypertension (yes/no), diabetes mellitus (yes/no), statin treatment (yes/no), apoA1 and apoB100 (both continuous). We additionally included prior AMI in an extended model. Further adjustments for B-vitamin treatment, eGFR, serum albumin or CRP had minimal influence on the results and were excluded in the final model (data not shown). Proportionality assumptions were evaluated by assessing the schoenfeld residuals.

Serum lipid parameters and Vit-A were categorized according to their median values and the effect modifications with plasma neopterin were tested by including interaction product terms in the Cox models. Survival according to the median values of neopterin and 
LDL-C or apoB100 or Vit-A was estimated using the Kaplan-Meier method and compared by the log-rank test. Potential nonlinear-interrelationships between plasma neopterin, serum LDL-C or apoB100 levels, and incident AMI was explored visually by plotting generalized additive models, adjusted for age and sex, and surface spline plots from the unadjusted hazards model.

All $\mathrm{P}$ values were 2 -tailed, and considered significant when $<0.05$. Statistical analyses were performed using SPSS (version 23 SPSS Inc, IBM, NY, USA) and R (version 3.1.2 R Core Team, Vienna, Austria)

\section{Patient and public involvement}

The current study was conducted without patient involvement. Study patients were not involved in research design, the outcome measures, data analysis, result interpretation or the writing of this manuscript. However, we do have plans to disseminate the results of the research to the relevant patient community. 


\section{Results}

\section{Baseline characteristics}

The median $\left(25^{\text {th }}-75^{\text {th }}\right.$ percentile $)$ age was $62(55-70)$ years, and $72 \%$ were men. Median (25th-75th percentile) plasma neopterin, LDL-C, apoB100 and Vit-A were 8.2 (6.7$10.4) \mathrm{nmol} / \mathrm{L}, 2.90(2.4-3.7) \mathrm{mmol} / \mathrm{L}, 0.87(0.73-1.04) \mathrm{g} / \mathrm{L}$ and $2.82(2.5-3.3) \mu \mathrm{mol} / \mathrm{L}$, respectively. As presented in Table 1, a higher proportion of patients in the upper neopterin quartile were older, women, had hypertension, diabetes mellitus or established coronary heart disease. We also observed positive associations of neopterin with CRP, Vit-A and highsensitivity troponin-T, whereas there were inverse associations with eGFR, serum albumin, as well as with current smoking. Plasma neopterin was also negatively associated with serum total cholesterol and LDL-C/apoB100 ratio, whereas no pronounced associations were observed with levels of LDL-C, apoB100, HDL-C, or apoA1. Furthermore, patients with higher neopterin levels were less likely to use statins, but were more frequently prescribed $\beta$ blockers, angiotensin-converting enzyme inhibitors or angiotensin receptor blockers 
Table 1. Baseline characteristics of the study population according to quartiles of plasma neopterin among patients with stable angina pectoris

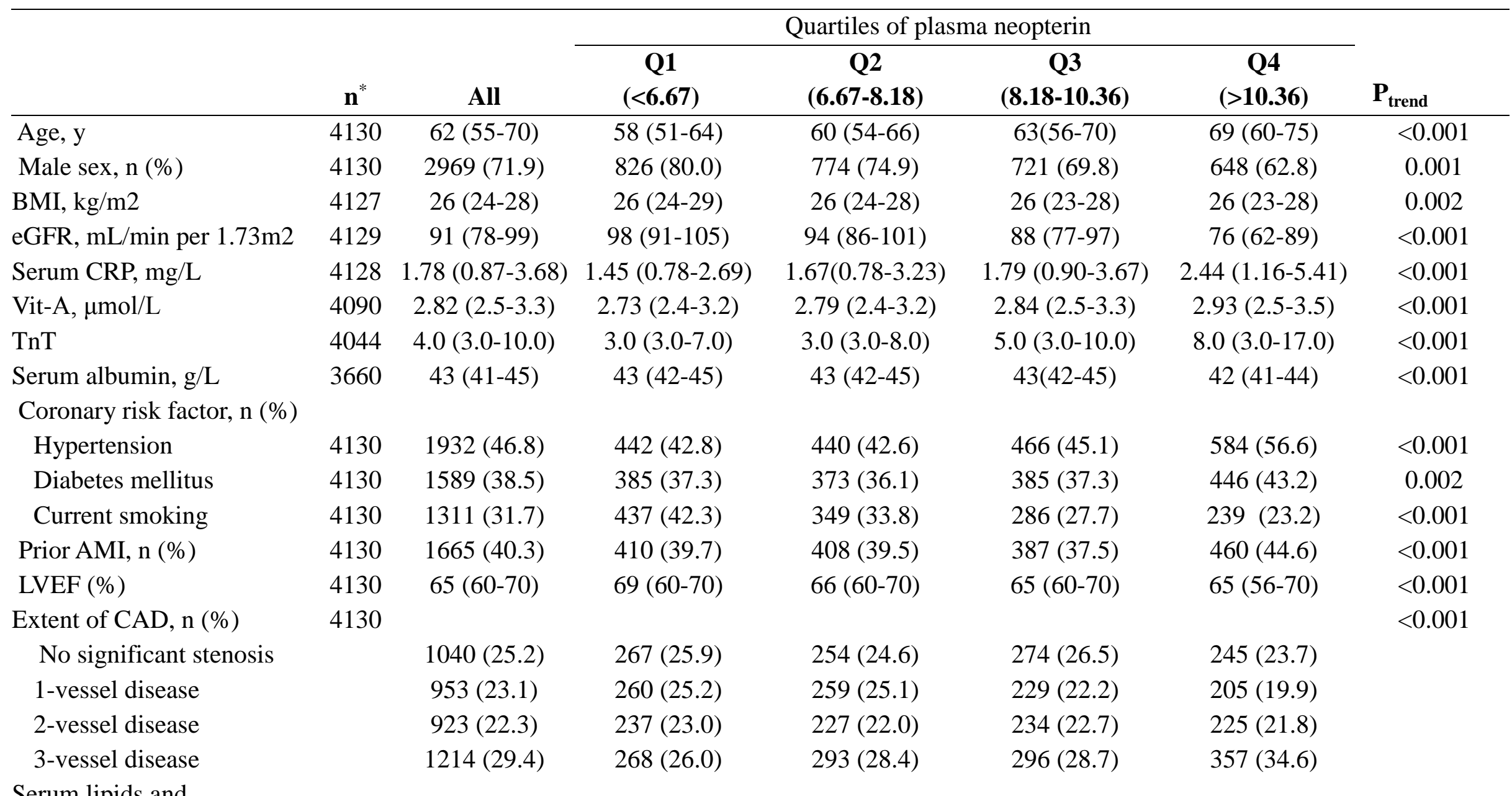


apolipoproteins

Total cholesterol, $\mathrm{mmol} / \mathrm{L}$

LDL-C, mmol/L

ApoB100, g/L

HDL-C, mmol/L

Apo A1, g/L

LDL-C/ApoB100, $\mathrm{mmol} / \mathrm{g}$

$4128 \quad 4.9(4.3-5.7) \quad 5.0(4.3-5.8)$

$4.90(4.3-5.7)$

$2.91(2.4-3.7)$

$5.0(4.3-5.7)$

$2.95(2.4-3.7)$

0.03

$\begin{array}{lll}4126 & 2.90(2.4-3.7) \quad 2.97(2.4-3.7)\end{array}$

$0.86(0.74-1.03)$

$0.86(0.72-1.04)$

$2.90(2.3-3.7)$

0.192

$41290.87(0.73-1.04) \quad 0.87(0.74-1.05)$

$1.20(1.0-1.5)$

$1.30(1.1-1.5)$

$0.87(0.73-1.05)$

$4129 \quad 1.20(1.0-1.50) \quad 1.20(1.0-1.5)$

$1.31(1.14-1.47)$

$1.31(1.14-1.51)$

$1.20(1.0-1.5)$

0.94

$41281.30(1.13-1.48) \quad 1.30(1.14-1.47)$

$41253.41(3.10-3.72) \quad 3.45(3.16-3.75) \quad 3.43(3.13-3.74) \quad 3.40(3.10-3.71) \quad 3.37(3.04-3.68)$

$41253.41(3.10-3.72) \quad 3.45(3.16-3.75) \quad 3.43(3.13-3.74) \quad 3.40(3.10-3.71) \quad 3.37(3.04-3.68)$

$1.27(1.11-1.47)$

0.48

0.863

0.005

$(\%)$

\begin{tabular}{lccccccc} 
Statin & 4119 & $3313(80.4)$ & $836(81.0)$ & $857(83.0)$ & $812(78.6)$ & $803(77.8)$ & 0.01 \\
$\beta$-Blocker & 4130 & $2993(72.5)$ & $739(71.6)$ & $752(72.8)$ & $720(69.7)$ & $782(75.8)$ & 0.02 \\
ACEIs and/or ARB & 4130 & $1318(31.9)$ & $279(27.3)$ & $288(27.9)$ & $329(31.8)$ & $422(40.9)$ & $<0.001$ \\
\multicolumn{5}{l}{ Continuous variables are presented as medians (25th-75th percentiles) and categorical variables as numbers (percentages). ACEI indicates }
\end{tabular}

Continuous variables are presented as medians (25th-75th percentiles) and categorical variables as numbers (percentages). ACEI indicates angiotensin-converting enzyme inhibitor; AMI, acute myocardial infarction; ApoA1, apolipoprotein A1; ApoB100, apolipoprotein B100; ARB, angiotensin receptor blocker; BMI, body mass index; CRP, C-reactive protein; eGFR, estimated glomerular filtration rate; HDL-C, high-density lipoprotein cholesterol; LDL-C, low-density lipoprotein cholesterol; LVEF, left ventricular ejection fraction; Tnt, Troponin T; Vit-A, Vitamin A measured as serum retinol.

* Patients with valid measurements 


\section{Plasma neopterin and incident AMI}

$530(12.8 \%)$ patients experienced an AMI during a median $\left(25^{\text {th }}-75^{\text {th }}\right.$ percentile $)$

follow-up time of 7.5 (6.3-8.7) years. Plasma neopterin was associated with increased risk of AMI in age and sex-adjusted model (HR [95\% CI] per 1-SD:1.26 [1.17-1.35]; $P<0.001)$

(Table 2 and Supplemental Figure 1). Multivariate adjustment (Table 2) or including prior AMI in the model 2 slightly attenuated the risk relationship.

Table 2. Risk association between plasma neopterin and incident acute myocardial infarction

\begin{tabular}{lcc}
\hline & HR $(95 \% \mathrm{CI})^{*}$ & P-value \\
\cline { 2 - 3 } Unadjusted & $1.33(1.25-1.42)$ & $<0.001$ \\
Model $1^{\dagger}$ & $1.26(1.17-1.35)$ & $<0.001$ \\
Model $2^{\ddagger}$ & $1.24(1.15-1.33)$ & $<0.001$ \\
Model 2+ prior AMI & $1.21(1.12-1.30)$ & $<0.001$ \\
\hline
\end{tabular}

*Per 1-SD increase in log-transformed concentrations.

$\dagger$ Model 1 adjusted for age, and sex

$\$$ Model 2 adjusted for age, sex, BMI, hypertension, diabetes mellitus , smoking, apolipoprotein A1, apolipoprotein B100 and statin treatment.

\section{The neopterin-AMI risk association according to serum lipid parameters and Vit-A}

Table 3 depicts risk estimates between plasma neopterin and AMI according to the subgroups of serum lipids and Vit-A. Among patients with below-median LDL-C levels, plasma neopterin showed a linear positive association with incident AMI (HR [95\% CI] per SD:1.35 [1.24-1.48]; $P<0.001)$, whereas no significant risk relationship was found among those with above-median LDL-C $\left(P_{\text {interaction }}=0.03\right)$ (Table 3, Figure 1 and Supplemental Figure 2). Similarly, there was a stronger relationship between plasma neopterin and AMI in patients 
with below compared to above-median serum apoB100 (Table 3, Figures 2 and Supplemental Figure 3) $\left(P_{\text {interaction }}=0.002\right)$. We also observed a particularly strong relationship of neopterin in patients with serum Vit-A levels above the median $\left(\mathrm{P}_{\text {interaction }}=0.03\right)$ (Table 3$)$.

Table 3. Associations between plasma neopterin (log transformed per SD) and AMI according to subgroups of prespecified lipid parameters and Vit-A

\begin{tabular}{|c|c|c|c|c|}
\hline Subgroups & Events/Total & $\mathrm{HR}(95 \% \mathrm{CI}) *$ & P-value & Pint \\
\hline \multicolumn{5}{|l|}{$\mathrm{LDL} \mathrm{C}^{\dagger}$} \\
\hline$\leq$ median & $263 / 2075$ & $1.35(1.24-1.48)$ & $<0.001$ & 0.03 \\
\hline$>$ median & $267 / 2051$ & $1.11(0.97-1.26)$ & 0.12 & \\
\hline \multicolumn{5}{|l|}{ ApoB100 ${ }^{\dagger}$} \\
\hline$\leq$ median & $253 / 2127$ & $1.42(1.27-1.58)$ & $<0.001$ & 0.002 \\
\hline$>$ median & $277 / 2002$ & $1.14(1.02-1.26)$ & 0.02 & \\
\hline \multicolumn{5}{|l|}{ HDL-C ${ }^{\dagger}$} \\
\hline$\leq$ median & $312 / 2123$ & $1.19(1.09-1.31)$ & $<0.001$ & 0.18 \\
\hline$>$ median & $218 / 2006$ & $1.36(1.18-1.57)$ & $<0.001$ & \\
\hline \multicolumn{5}{|l|}{ ApoA $1^{\dagger}$} \\
\hline$\leq$ median & $303 / 2107$ & $1.21(1.10-1.32)$ & $<0.001$ & 0.20 \\
\hline$>$ median & $227 / 2021$ & $1.32(1.16-1.50)$ & $<0.001$ & \\
\hline \multicolumn{5}{|l|}{ LDL-C/ApoB100 } \\
\hline \multicolumn{5}{|l|}{$\operatorname{ratio}^{\dagger}$} \\
\hline$\leq$ median & $292 / 2066$ & $1.27(1.17-1.39)$ & $<0.001$ & 0.47 \\
\hline$>$ median & $238 / 2059$ & $1.19(1.03-1.36)$ & 0.02 & \\
\hline \multicolumn{5}{|l|}{$\mathrm{Vit} \mathrm{A}^{\dagger}$} \\
\hline$\leq$ median & $267 / 2043$ & $1.11(0.97-1.28)$ & 0.13 & 0.03 \\
\hline$>$ median & $254 / 2047$ & $1.32(1.21-1.44)$ & $<0.001$ & \\
\hline
\end{tabular}

AMI indicates acute myocardial infarction; ApoA1, apolipoprotein A1; ApoB, apolipoprotein B; HDL-C, high-density lipoprotein cholesterol; LDL-C, low-density lipoprotein cholesterol; Vit-A, vitamin A measured a serum retinol *Adjusted for age and sex $\dagger$ Patients with valid measurements $=4126$ (in LDL-C analyses), 4129 (ApoB100 analyses), 4129 (HDL-C analyses), 4128 (ApoA1 analyses), 4125 (LDL-C/ApoB100 ratio analyses) and 4090 (Vit-A analyses). 
Accordingly, reduced survival was observed in patients with a high concentration of neopterin but low concentration of LDL-C or apoB100 (P<0.001) (Supplemental Figures 4 and 5). This was also the case in patients with high concentrations of both neopterin and VitA $(\mathrm{P}<0.001)$ (Supplemental Figure 6). The effect modification by LDL-C or apoB100 and Vit-A subgroups were essentially similar after multivariate adjustment (Supplemental Table 1). The neopterin-AMI association was not significantly modified by serum HDL-C or apoA1 (Table 3 and Supplemental Table 1).

Notably, the neopterin-LDL-C or apoB100 risk associations were even stronger among patients receiving statin treatment after angiography (Supplemental Table 2 through 4) (both, $\mathrm{P}$ for 3-way-interaction $\leq 0.03$ ). 


\section{Discussion}

In patients undergoing coronary angiography for suspected SAP, plasma neopterin predicted the risk of incident AMI predominantly in statin-treated patients with lower serum LDL-C or apoB100 concentrations, as well in patients with elevated Vit-A.

\section{Neopterin, lipid metabolism, and cardiovascular disease}

Our findings of an increased cardiovascular risk associated with higher plasma neopterin is concordant with previous research[6-8], including SAP[6] and may underline the importance of immune cell activation in the development of atherogenesis. The LDL-C hypothesis identifies LDL-C as a causal factor of atherosclerotic CVD [2]; however, there remains some uncertainty about the direct role of LDL-C [21]. On the other hand, data regarding the association between Vit-A and cardiac events have been contradictory[22]. To our knowledge, this is the first large-scale investigation to demonstrate that serum LDL-C, apoB100 and Vit-A concentrations modifies the association of neopterin with cardiovascular outcomes.

\section{Possible mechanisms and implications}

We observed no significant associations of plasma neopterin with serum LDL-C or apoB100 at baseline; however, the putative associations may be masked by statin therapy, as more subjects in the lower neopterin quartiles were prescribed statins, which is in line with a previous report[7]. The activation of monocytes into macrophages accumulating intracellular cholesterol and other lipids in the subendothelial layer of arteries is a hallmark for atherosclerosis, and is also fundamental for the concept that inflammation is a promoting factor at all stages of atherosclerosis[1]. Here, we propose that the current findings may be related to the impaired hepatic VLDL production, monocyte/macrophage activation, and the metabolic cross-talk of these interconnected pathways. 
Dysfunctional VLDL secretion has been associated with increased hepatic lipid accumulation $[11,12]$ and secretion of large VLDL particles with reduced cholesterol content, which leads to the formation of sdLDL particles with increased susceptibility to oxidative modification [12-14]. oxidized LDL activates monocytes[1, 15], and cholesterol oxidation products i.e oxysterols stimulate production of the active Vit-A form, all-trans retinoic acid [16], which again induces monocyte differentiation[17]. These findings may indicate the involvement of impaired hepatic apoB-containing lipoprotein secretion in monocytes/macrophages activation, reflected by neopterin production. Accordingly, we observed associations of neopterin with decreased LDL-C/apoB ratio and increased Vit-A, which represents the presence of sdLDL-particles[23] and modified cholesterol product[16], respectively. Activated monocytes take up modified LDL via the scavenger receptor, produces extra cholesterol and export free cholesterol to HDL-C. Notably, this export is regulated by ATP-binding cassette transporter A1, which is stimulated by both neopterin[18] and all-trans retinoic acid [24]. Thus, oxidized LDL, increased Vit-A and neopterin production may primarily be a response and feed-back mechanism due to an impaired liver VLDL-C export, aiming to restore this deficiency of cholesterol in VLDL/LDL. Recently, in the same current cohort[25], we showed that that low apo A1, the primary protein constituent of HDL, was related to incident AMI primarily in those with high Vit-A levels. This may suggest that apoA1 deficiency and low HDL primarily is related to the risk of atherosclerosis when the endothelium produces oxidized LDL as a signal of impaired LDL-C supply. This hypothesis is further supported by our current observation, indicating that that the risk of AMI is particularly high when there are two signals of excess oxidized cholesterol production, i.e., the combination of elevated Vit-A and neopterin. Notably, the neopterin related risk association was stronger in those with low LDL-C or apoB100, and these findings thus 
strengthen the hypothesis that an impaired cholesterol supply from the liver to the endothelium may promote atherosclerosis.

The mechanism responsible for reduced hepatic VLDL output is not known but could be related to high endogenous PPAR $\alpha$ activity in the liver. Treatment with PPAR $\alpha$ agonist has been demonstrated to increase catabolism of apoB100[9], which is a ligand for LDL-receptormediated endocytosis of LDL particles, in addition to being a crucial component of VLDL synthesis and assembly[14]. Additionally, PPAR $\alpha$ agonism attenuates GNMT transcription [10], a critical regulator of VLDL export[11]. In line with this, recent animal data suggest that PPAR $\alpha$ activation may promote hepatic steatosis[26]. Notably, we observed a strong relationship between neopterin and AMI among patients with low LDL-C or ApoB100, and even more so in those treated with statins, which is suggested to stimulate hepatic PPAR $\alpha$ gene expression and activation[3]. This suggests the involvement of curtailed lipid flux between the liver and peripheral tissues due to excess hepatic PPAR $\alpha$ activation in neopterinrelated atherogenesis. Lowering of LDL-C levels by statin-treatment has been shown to result in CVD reduction[2] and these findings, at first glance, may, therefore, seem discordant with the current analysis. We do not know the initial lipid levels of these patients as the majority were already treated with statins at the time of angiography; however, cardiovascular events are common even in subjects attaining low LDL-C concentrations[4]. Although it is difficult to draw firm conclusions about particular mechanisms based on one study alone, our findings may, however, provide some insight into the residual CVD risk despite treatment with lipidlowering statins[4].

\section{Strengths and limitations}

The strengths of our study include the large and well-characterized sample and its prospective design with long-term follow-up. 
There are limitations to our study. First, the follow-up was ascertained from the patient administrative data; hence, we cannot rule out some underreporting or other misclassification of clinical endpoints. However, we find it unlikely that such misclassification differs according to levels of plasma neopterin or serum lipid parameters. Second, some factors were not assessed, especially malnutrition which can potentially influence the levels of lipids [27]. However, we do not suspect any confounding role of nutrition because adjusting for serum albumin, a sensitive marker of undernutrition[27], and CRP, a measure of systemic inflammation[27], the interactions remained unchanged. Fourth, high neopterin production is associated with increased oxidative stress[5]. Intriguingly, despite the occurrence of oxidative stress in smokers[1], we observed an inverse association between plasma neopterin and smoking status. This finding is in line with a previous report[28] and the relationship may be explained by the fact that tobacco smoke has been suggested to attenuate the human immune system via suppressing T-helper-type 1 lymphocytes[29]. Third, nearly $46 \%$ of the patients received treatments with folic acid plus vitamin B12 or B6, and thus our results may not be generalizable to untreated populations. However, supplementation with B-vitamins is reported to have no significant effects on plasma neopterin levels [30]. Moreover, interactions persisted after adjusting for B-vitamin treatment. Finally, the mechanism detailed in our study are purely speculative, and additional investigations are thus needed to better explore the role of low lipid measures and statin therapy in neopterin-related atherogenesis.

\section{Conclusion}

In patients with suspected SAP, the increased risk of AMI associated with elevated neopterin levels was predominately present in patients with high serum Vit-A, and those with low circulating LDL-C or apoB100 levels, in particular among whom receiving statin therapy after angiography. It, therefore, seems conceivable that increased levels of neopterin and low 
serum LDL-cholesterol and apoB100 may share some common pathophysiological mechanisms that contribute to the pathogenesis of atherosclerotic CVD, which might include impaired hepatic VLDL-C output. Our findings motivate further investigations on the relationship between lipid metabolism and cellular immune activation, and atherothrombosis.

\section{Acknowledgements}

The authors thank all the WENBIT coworkers at Haukeland and Stavanger university hospitals, as well as the laboratory person for biochemical analyses at Bevital A/S, and the Lipid research group, University of Bergen, Bergen, Norway. We are grateful to Tomislav Dimoski at the Norwegian Institute of Public Health, Norway, for his contribution by developing the software necessary for obtaining admission data from Norwegian public hospitals and conducting data collection and quality assurance of data in this project.

\section{Data sharing}

The data will be made available to other researchers for purposes of reproducing the results or replicating the procedure upon reasonable request.

\section{Contributors}

OKN and ID designed research; ID analyzed the data, performed analysis, wrote the manuscript and had primary responsibility for the final content; ID, SS, ERP, GFTS, VL, TO, DWN, JEN, ØM, PMU, GST and OKN conducted research. All authors critically reviewed and revised the manuscript. $\mathrm{OKN}$ and ID are the guarantors

\section{Funding}


Funding was provided by the University of Bergen, the Department of Heart Disease at Haukeland University Hospital, the Western Norway Regional Health Authority, and the Foundation to Promote Research into Functional Vitamin B12 Deficiency, Bergen, Norway.

\section{Copyright/license for publication}

The Corresponding Author has the right to grant on behalf of all authors and does grant on behalf of all authors, an exclusive licence (or non exclusive for government employees) on a worldwide basis to the BMJ Publishing Group Ltd and its Licensees to permit this article (if accepted) to be published in HEART editions and any other BMJPGL products to exploit all subsidiary rights"

\section{Disclosures}

None.

\section{References}


1. Singh RB, Mengi SA, Xu YJ, et al. Pathogenesis of atherosclerosis-A multifactorial process. Exp Clin Cardiol 2002;7:40-53.

2. Ference BA, Ginsberg HN, Graham I, et al. Low-density lipoproteins cause atherosclerotic cardiovascular disease. 1. Evidence from genetic, epidemiologic, and clinical studies. A consensus statement from the European Atherosclerosis Society Consensus Panel. Eur Heart J 2017;38:2459-72.

3. Seo M, Inoue I, Ikeda M, et al. Statins activate human PPARalpha promoter and increase PPARalpha mRNA expression and activation in HepG2 cells. PPAR Res 2008;2008:316306.

4. Sampson UK, Fazio S, Linton MF. Residual cardiovascular risk despite optimal LDL cholesterol reduction with statins: the evidence, etiology, and therapeutic challenges. Curr Atheroscler Rep 2012;14:1-10.

5. Murr C, Widner B, Wirleitner B, et al. Neopterin as a marker for immune system activation. Curr Drug Metab 2002;3:175-87.

6. Pedersen ER, Midttun $\varnothing$, Ueland PM, et al. Systemic markers of interferon- $\gamma$-mediated immune activation and long-term prognosis in patients with stable coronary artery disease. Arterioscler Thromb Vasc Biol 2011;31:698-704.

7. Grammer TB, Fuchs D, Boehm BO, et al. Neopterin as a predictor of total and cardiovascular mortality in individuals undergoing angiography in the Ludwigshafen risk and cardiovascular health study. Clin Chem 2009;55:1135-46.

8. Sulo G, Vollset SE, Nygård O, et al. Neopterin and kynurenine-tryptophan ratio as predictors of coronary events in older adults, the Hordaland Health Study. Int J Cardiol 2013;168:1435-40.

9. Shah A, Rader DJ, Millar JS. The effect of PPAR-alpha agonism on apolipoprotein metabolism in humans. Atherosclerosis 2010;210:35-40 
10. Sheikh K, Camejo G, Lanne B, et al. Beyond lipids, pharmacological PPARalpha activation has important effects on amino acid metabolism as studied in the rat. Am J Physiol Endocrinol Metab 2007;292:E1157-65.

11. Liao Y, Chen T, Lee T, et al. Glycine N-methyltransferase deficiency affects NiemannPick type C2 protein stability and regulates hepatic cholesterol homeostasis. Mol Med 2012;18:412-22.

12. Fujita K, Nozaki Y, Wada K, et al. Dysfunctional very-low-density lipoprotein synthesis and release is a key factor in nonalcoholic steatohepatitis pathogenesis. Hepatology 2009;50:772-80.

13. Adiels M, Taskinen MR, Packard C, et al. Overproduction of large VLDL particles is driven by increased liver fat content in man. Diabetologia 2006;49:755-65.

14. Brea A, Mosquera D, Martin E, et al. Nonalcoholic fatty liver disease is associated with carotid atherosclerosis: a case-control study. Arterioscler Thromb Vasc Biol 2005;25:1045-50.

15. Frostegard J, Nilsson J, Haegerstrand A, et al. Oxidized low density lipoprotein induces differentiation and adhesion of human monocytes and the monocytic cell line U937. Proc Natl Acad Sci USA 1990,87:904-8.

16. Huq MD, Tsai NP, Gupta P, et al. Regulation of retinal dehydrogenases and retinoic acid synthesis by cholesterol metabolites. EMBO J 2006;25:3203-13.

17. Hemmi H, Breitman TR. Induction of functional differentiation of a human monocytic leukemia cell line (THP-1) by retinoic acid and cholera toxin. Jpn J Cancer Res 1985;76:345-51.

18. Shirai R, Sato K, Yamashita T, et al. Neopterin counters vascular inflammation and atherosclerosis. J Am Heart Assoc 2018;7:e007359. 
19. Svingen GF, Ueland PM, Pedersen EK, et al. Plasma dimethylglycine and risk of incident acute myocardial infarction in patients with stable angina pectoris. Arterioscler Thromb Vasc Biol 2013;33:2041-8.

20. Sulo G, Igland J, Vollset SE, et al. Cardiovascular disease and diabetes mellitus in Norway during 1994-2009 CVDNOR — a nationwide research project. Nor Epidemiol 2013;23:101-7.

21. Ravnskov U, de Lorgeril M, Diamond DM et al. LDL-C Does Not Cause Cardiovascular Disease: a comprehensive review of current literature. Expert Rev Clin Pharmacol 2018;11:959-70.

22. Olsen T, Blomhoff R. Retinol, Retinoic Acid, and Retinol-Binding Protein 4 are Differentially Associated with Cardiovascular Disease, Type 2 Diabetes, and Obesity: An Overview of Human Studies. Adv Nutr Published Online First: 23 December 2019. doi: 10.1093/advances/nmz131.

23. Kaneva AM, Potolitsyna NN, Bojko ER. Usefulness of the LDL-C/apoB ratio in the overall evaluation of atherogenicity of lipid profile. Arch Physiol Biochem 2017;123:16-22.

24. Wagsater D, Dimberg J, Sirsjo A. Induction of ATP-binding cassette A1 by all-trans retinoic acid: possible role of liver X receptor-alphaInt. J Mol Med 2003;11:419-23.

25. Olsen T, Vinknes KJ, Svingen GF, et al. Cardiovascular disease risk associated with serum apolipoprotein B is modified by serum vitamin A. Atherosclerosis 2017;265:325-30.

26. Yan F, Wang Q, Xu C, et al. Peroxisome proliferator-activated receptor $\alpha$ activation induces hepatic steatosis, suggesting an adverse effect. PLoS One 2014;9:e99245. 
27. Hrnciarikova D, Hyspler R, Vyroubal P, et al. Serum lipids and neopterin in urine as new biomarkers of malnutrition and inflammation in the elderly. Nutrition 2009;25:303-8.

28. Diamondstone LS, Tollerud DJ, Fuchs D, et al. Factors influencing serum neopterin and beta 2-microglobulin levels in a healthy diverse population. J Clin Immunol 1994;14:368-74.

29. Moszczynski P, Zabinski Z, Moszczynski P, et al. Immunological findings in cigarette smokers. Toxicol Lett 2001;118:121-7.

30. Bleie $\varnothing$, Semb AG, Grundt H, et al. Homocysteine-lowering therapy does not affect inflammatory markers of atherosclerosis in patients with stable coronary artery disease. J Intern Med 2007;262:244-53. 
Figure Legends

Figure 1. The risk association between log-transformed neopterin and acute myocardial infarction in subgroups of serum LDL-C levels, adjusted for age and sex. Shaded areas around the curves depict 95\% CI. Kernel density plots show the distribution of plasma neopterin. LDL-C indicates low-density lipoprotein cholesterol.

Figure 2. The age and sex adjusted risk association between log-transformed neopterin and acute myocardial infarction according to subgroups of apoB100 levels. Shaded areas around the curves depict $95 \%$ CI. Kernel density plots are superimposed along the $\mathrm{x}$-axis, to display the distribution of plasma neopterin. apoB100 indicates apolipoprotein B100 